\title{
On-road Vehicle Tracking using Laser Scanner with Multiple Hypothesis Assumption
}

\author{
Kyungjin Ryu, Seongkeun Park, Jae Pil Hwang, Euntai Kim and Mignon Park \\ School of Electric and Electronic Eng. Yonsei Univ.
}

\begin{abstract}
Active safety vehicle devices are getting more attention recently. To prevent traffic accidents, the environment in front and even around the vehicle must be checked and monitored. In the present applications, mainly camera and radar based systems are used as sensing devices. Laser scanner, one of the sensing devices, has the advantage of obtaining accurate measurement of the distance and the geometric information about the objects in the field of view of the laser scanner. However, there is a problem that detecting object occluded by a foreground one is difficult. In this paper, criterions are proposed to manage this problem. Simulation is conducted by vehicle mounted the laser scanner and multiple-hypothesis algorithm tracks the candidate objects. We compare the running times as multi-hypothesis algorithm parameter varies.
\end{abstract}

Key Words : Active safety systems, laser scanner, occlusion, multi-hypothesis, multi-target tracking.

\section{Introduction}

In the future, active safety systems for vehicles are getting more importance[1-4]. For active safety systems, the environment in front and even around the vehicle must be observed and the robust algorithm must be applied to detect, classify, track the objects and predict its the next behavior. In recent applications, camera and radar are used as the main sensing devices. Although the laser scanner has a high price, it offers the advantage of obtaining accurate distance and geometric information about the objects in front of the vehicle.

Radar provides distance information by emitting modulated pulsed HF-radiation. The relative radial position and velocity of objects is detected using the time-of-flight principle and the Doppler Effect. The main disadvantage of radar sensors used today for vehicles is their limited angle of view because the antenna of the radar is fixed. Moreover, they have a poor angle resolution. The typical angle of view of radar is 12 degrees and the angular resolution is less than 4 degrees. Improved radar systems are currently under development. However, as the fundamental limitation is caused by the fixed position of the antennas, comparatively small improvements can be expected. Although multiple objects can be tracked using radar sensors, the precise geometric interpretation is impossible [5]. Our goal is to make a system that can be used for high speed situation, approximately $60 \mathrm{~km}$ to $80 \mathrm{~km}$. To be able to cope with this situation, we need to cover long range up to $100 \mathrm{~m}$. To meet this criteria, laser scanner is the best solution. Many works have been done on vehicular applications of laser scanners.

Manuscript received Dec. 14, 2007; revised Jun. 12, 2008. This work was supported by grant number R01-2006-00011016-0. from Basic Research Program of the Korea Science \& Engineering Foundation.
Fuestenberg[6] describes capabilities for pedestrian classification and correctly segmenting objects in the presence of occlusion, but gives little implementation detail. The odometry motion model is used to estimate the self-motion, removing the kinematic effects of sensor motion. A Kalman filter is used for velocity estimation. Tracks are classified as vehicle, pedestrian and etc., using apparent shape and behavior over time. Streller[7] describes a similar system with additional details. The Kalman filter estimates motion based on the position change of the estimated track's center-point. The object classification is used to fit a class-specific prior rectangular model to the points. Although the details are not explained, it appears to be an approach to reduce the shape-change motion artifacts. This success would depend on the correctness of the classification and the prior model. Each object classes also has distinct fixed Kalman filter parameters. A multi-hypothesis approach is used to mitigate the effect of the classification error [8].

Streller[9] proposed a new method using a multi-hypothesis approach. This approach keeps the tracks of all feasible combinations of separated objects. It also allows various classification results. Thus, it is possible to track several versions of objects. By this reason, no feasible objects will be unregarded. In association process, Streller used the hypothesis dependencies matrix [10]. In addition, Streller also simulated using multilayer laser scanner [11].

MacLachlan used the linear feature tracking to handle the shape change problem. In the segmentation process, two objects that are apparently one object that divided by a foreground object are combined as one object, but how to combine two objects is not mentioned in detail. To reduce the effect of occlusion, Streller used the multi-hypothesis in segmentation, classification and tracking. Multiple hypothesis approach is robust. However, it takes much time and needs much resources. Therefore, Streller method is not suitable for implementation in real-time.

These previous works had a huge contribution in this 
field. But they still have some limitations. Data measurement from the laser scanner includes lots of noise and occlusion. In this paper, we propose a novel method to filter and track the objects with this noise and occlusion of present situation. Our method is based on the geometrical analysis of the raw data from the laser scanner and Multi-hypothesis algorithm which is used to track the candidate objects.

Simulation is conducted on the data that is gained from the laser scanner mounted on a test vehicle. We compare the running times of multi-hypothesis algorithm with various parameters to find parameter that can be used for real-time implementation. We implemented the Obata's multi-hypothesis tracking that reduces the computation time using N-Best algorithm. It is applied to the track of objects to reduce the running time[12].

The paper is organized as follows. In Section 2, the process to detect the vehicles is presented with the handling method of noise and occlusion. In Section 3, we briefly cover the multi-hypothesis algorithm. In Section 4, simulation results are given to show the result of multi-target tracking and running time as parameter of multi-hypothesis varies. Finally, the conclusion is drawn in Section 5.

\section{Detecting vehicles}

Before tracking the object, Laser Scanner data needs to be classified into objects. The raw Laser Scanner data consists of many points. Tracking all points needs very expensive computation load and it is not efficient. Because one object is composed of many points, tracking one object produces similar result that tracking entire points of the object does.

The data in each scan is not always reliable. Zero or maximum value is sometimes received from Laser Scanner, so we check the data before segmentation. The data from $0.1 \mathrm{~m}$ to $100 \mathrm{~m}$ range is reliable and that of other range is assumed as noise. In noise case, -1 is inserted to range to discriminate reliable data because range value is always greater than 0 .

$$
\begin{cases}\text { Range }^{\prime}=\text { Range } & 0.1 \mathrm{~m}<\text { Range }<100 \mathrm{~m} \\ \text { Range }^{\prime}=-1 & \text { Otherwise }\end{cases}
$$

\subsection{Criterion 1: Noise handling}

One object can be measured from several angles depending on Its pose. From the lidar data, several sequential measurement points appear to build the shape of one object. The first criterion is to divide the points in the sequence into individual objects by distance between each points. Fig 2 shows the distance between adjacent points. Intuitively, we think the points that from $\mathrm{P} 0$ to $\mathrm{P} 4$ are one object and the points that from P5 to P8 are another object. $d_{i}$ is the distance of adjacent points between $p_{i}$ and $p_{i+1}$. If $d_{i} \leqq$ Threshold, then the points $p_{i}$ and $p_{i+1}$ are same object.

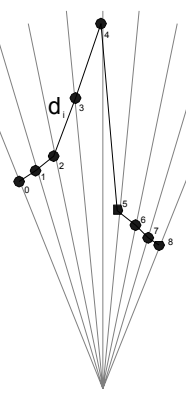

Fig. 1 Distance between adjacent points

The threshold is not a constant value. The distance between adjacent points is different as range changes. The threshold is determined by the distance of adjacent point in front of laser scanner at angle theta.

$$
\begin{aligned}
& \angle \Delta \text { is } 0.25^{\circ}, \text { resolution of laser scanner } \\
& b \approx r \angle \Delta \\
& \angle a=90.125^{\circ} \approx 90^{\circ}
\end{aligned}
$$

We can approximate this triangle to a right-angled triangle in Fig. 2 (b). And threshold given as

$$
\text { Threshold } d_{i}=\frac{b}{\cos \angle \theta}=\frac{r \angle \theta}{\cos \angle \theta} \text {. }
$$

For this simulation we set the threshold

$$
\text { Threshold } d_{i}=\frac{b}{\cos \angle \theta}+C \text {. }
$$

$\mathrm{C}$ is constant $(\mathrm{C}=0.2)$ and $\theta$ is $85^{\circ}$.

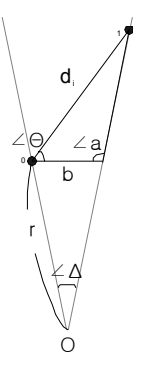

(a) Adjacent points

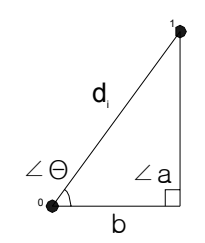

(b) Approximation to a right-angled triangle
Fig. 2 Approximation of threshold

Because the sequence includes false data, the threshold value needs to be modified. When false data is included, the distance between adjacent points becomes longer. Fig. 3 shows the laser scanner data including false data. P2, P6, and P7 is false data that is thrown away. In this simulation, we don't use the threshold just by multiplied by absent data numbers. It's because threshold grows as absent 
data number increases. So threshold redefined as

$$
\text { Threshold } d_{i}=\frac{(r+k) \angle \theta}{\cos \angle \theta}+C \text {. }
$$

where $\mathrm{k}$ is the number of absent data. From our repeated simulation with various parameters, we limit the absent number under 5. Otherwise, different others might be combined to one.

If the object consists of 4 points or less, it is hard to determine whether the object is real object or noise. So the object under 4 points is deleted.

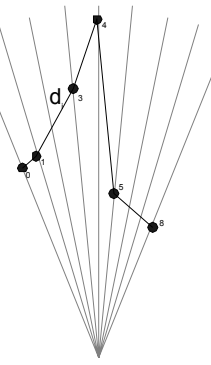

Fig. 3 Absent data in the laser scanner data

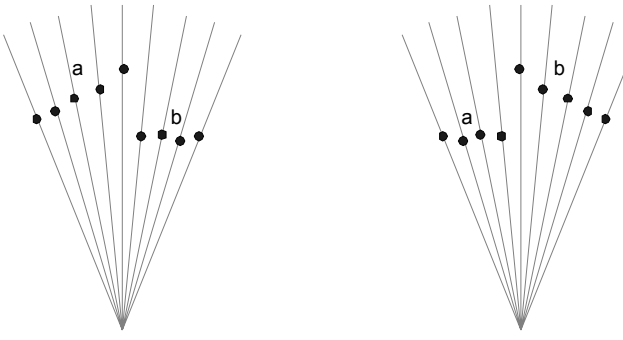

(a) Continue a, terminate b (b) Terminate a, continue b,

Fig. 4 Continue points or terminate points

\subsection{Criterion 2: Occlusion handling}

After Criterion 1, we can observe that one object may split into several objects. It is caused by noise or small object in font of large object. Removing these noises or small objects in front of a large object is a solution to this problem. Before removing, we must determine whether the object is continued or terminated. In Fig. 4 (a), object a is occluded by object $b$. In this case. the state of object a is set to continuation and that of object $b$ is set to termination. In Fig. 4 (b), vice versa.

We set some vague distance to determine the continuation or the termination of the object. If the distance difference of two objects is within vague distance, two objects are said to be continuous. In case of the beginning point of the segment being continuous, we check the prior segment's state whether it is also continuous. If the prior segment's state is termination, comparing is undone. When comparing two segmentations, just comparing the start point of current segment and the end point of the previous may be influenced by noise. To overcome this, we need to compare several points that is near to the beginning and the end of each segment. The number of comparison that needs to be done is determined as follow.

$$
N_{o}=\min (\# \text { of segmentation point }+3,10)
$$

\subsection{Criterion 3: Shape determination}

We are only interested in vehicles. Others that are not classified as a vehicle are not considered. It is better if others are not detected because it takes less time to track the object in multi-hypothesis algorithm. The feature of vehicle Laser Scanner data is shown in Fig. 5. We can conclude that vehicle is detected by the least square line fitting. Vehicle is represented by line one or two.

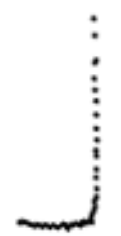

(a) Type I

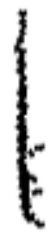

(b) Type II

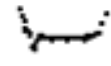

(c) Type III
Fig. 5 Feature of vehicle laser scanner data

\section{Multi-hypothesis tracking}

Fig. 6 gives the overview of multi-hypothesis tracking method. Hypotheses are formed as data received. Hypotheses consist of tracks whose state estimates are updated, usually with standard Kalman filtering techniques. As new data is received, the state estimation and covariance are used to form gates. The unlikely hypotheses are not made and updated. In addition to the hypothesis evaluation, management techniques (such as pruning, combining, and clustering) are also required to limit the number of hypotheses and to avoid the hypotheses from growing exponentially.

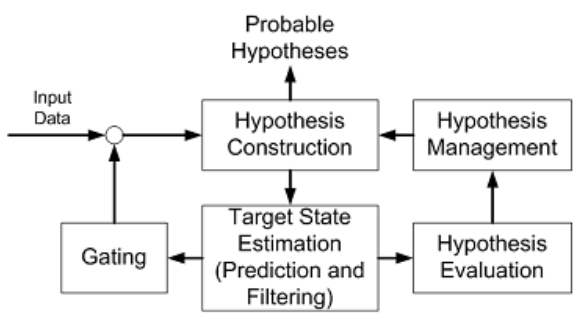

Fig. 6 Overview of multi-hypothesis tracking algorithm

Clustering divides the measurement to several small areas which do not include each other. Clustering makes the hypothesis to become several small problems. And set hypothesis in a cluster have limit numbers. That avoids hypothesis from growing exponentially.

$\mathrm{N}$-scan back memory sets the memory to limit its size. The branch that has the highest probability survives. The objective of the N-best algorithm is to find the best hy- 
pothesis in sequence. It does not make all the combination of the hypotheses and the measurements. This algorithm helps to reduce time consumption greatly.

\section{Simulation and Results}

In this paper the raw data from the laser scanner is obtained in a highway by a test vehicle shown in Fig. 7. Simulation is performed by $\mathrm{C}^{++}$program. The Laser Scanner is SICK's LD OEM. The LD OEM was mounted in the center of the frontal area of a test vehicle. SICK 's LD OEM is shown in Fig. 1-1. The angle range of the LD OEM covers up to 360 degrees. The angular resolution is $0.125^{\circ}$ up to $1.0^{\circ}$ (depending on the scan frequency). The scan frequency is $5 \mathrm{~Hz}$ up to $20 \mathrm{~Hz}$. The range of the LD OEM depends on the size and reflectivity of the target. The measurement range covers the nearly up to $250 \mathrm{~m}$ on reflecting targets. But it is hard to detect objects out of $100 \mathrm{~m}$ in real situation. In this paper, the LD OEM parameters are set as follows. The field of view of the laser scanner is set to $180^{\circ}$, angle step resolution is $0.25^{\circ}$, Rotation frequency of the scanner head is $5 \mathrm{~Hz}$.

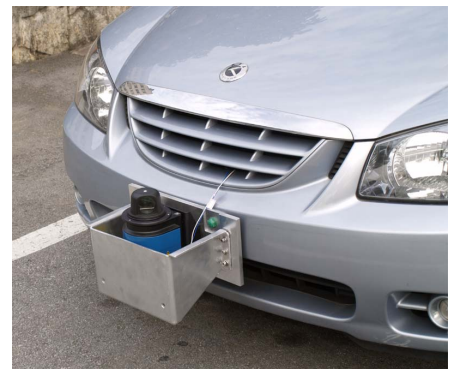

Fig. 7 Simulation vehicle with LD OEM

Table 1. Running time. Column is max hypothesis in cluster - max hypothesis in N-best. Row is N-scan back memory number. (sec.)

\begin{tabular}{|c|c|c|c|c|}
\hline & $2-1$ & $4-2$ & $8-4$ & $16-4$ \\
\hline 4 & 0.13 & 0.22 & 0.35 & 0.57 \\
\hline 5 & 0.16 & 0.39 & 0.71 & 1.16 \\
\hline 6 & 0.18 & 0.57 & 1.06 & 2.06 \\
\hline
\end{tabular}

Fig. 8 shows the results of the detected vehicles using shape analysis of raw data in the sequence scans. The center of half circle is position of laser scanner located. One grid is $8 \mathrm{~m}$. Small black points mean the raw laser scanner data. Different object has different colors. Detecting algorithm is performed for each scan, same object has different color in the next scan. Sometimes shape of vehicles is distorted because vehicle is moving. In that case, detecting algorithm can not detect the object.

Fig. 9 shows the results of the tracking detected objects using multi-hypothesis in sequence of scans. Each hypothesis uses the Kalman filter to update and correct process. Also, small black points mean the raw laser scanner data.
Other colors mean the different objects. In this result, same object has the same color in the next scan. The same color line appending to object is trajectory of object. In this simulation, we use $6 \mathrm{~N}$-back scan memory.

Table 1 shows the running time of entire algorithm as multi-hypothesis parameter varies. Running time is the mean of conducting algorithm running time in 1000th scan. Parameter values is low, and algorithm takes shorter time than vice versa. So, we need the parameter values that has running time under $0.25 \mathrm{~s}$ to simulate in real-time.

\section{Conclusions}

In this paper, simulation is conducted by the data from a vehicle mounted laser scanner. Analysis of the raw data from laser scanner is done geometrically and we used multiple-hypothesis algorithm to track the candidate objects. We compare the running times of multi-hypothesis algorithm with various parameters to find the optimal parameter that can be simulate in real time. We implemented the Obata's multi-hypothesis tracking that reduces the computation time using N-Best algorithm which is applied to track the objects which are for reducing the running time.

We can detect the vehicle from raw data using the segmentation and the shape procedures by the geometrical analysis due to high resolution of laser scanner. But, detecting vehicles from geometric image is still a challenging problem because of occlusion, which separation of objects into smaller pieces happens due to false measurement or small object in front of the object. This makes vehicle to be misclassified.

Detected objects have different kinematic behaviour. Tracking with multi-hypothesis gives robust performance. On the other hand, it takes much time and need much resources. Because of this reason, it is hard to run in real-time. Obata method that helps to reduce the running time using N-best algorithm and adjusting the parameter can run in real-time. However, as parameter value is lower, performance is worse. Detecting the vehicles within the sequence of scans inner road area is needed to reduce the input of multi-hypothesis tracking for robust performance and real-running time. We will work on these problems.

\section{Reference}

[1] D. H. Kim, "Multi-vehicle Route Selection Based on an Ant System," IJFIS, vol.8, no.1, pp. 61 67, 2008.

[2] D.-Y. Im, Y.-J. Ryoo, Y.-Y. Jung, J. Lee, Y.-H. Chang, S.-H. Kim and J.-H. Park, "Development of Autonomous Vehicle Using Array Magnetic Sensor," ISIS 2007 Proc. of 8th Symp. on Adv. Intel. Sys., pp. 914 918, 2007.

[3] E.-K. Lim, Y.-J. Kim, D.-S. Kim and K.-B. Kim, "Recognition of Car License Plates using Morphological Information and SOM Algorithm,", ISIS 
2003 Proc. of 4th Intern. Symp. on Adv. Intel. Sys., pp. 648 651, 2003.

[4] K. Nishimori, M. Okada, N. Ishihara and H. Tokutaka, "Vision System for Fuzzy Driving Control of an Automatic Vehicle," Proc. of 3rd Intern. Conf. on Fuzzy Logic, Neural Nets and Soft Comp., pp. 231 232, 1994.

[5] J. Sparbert, K. Dietmayer, D. Streller, "Lane detection and street type classification using laser range images," Proc. Intelligent Transportation Systems ,IEEE, pp. 454 459, 2001.

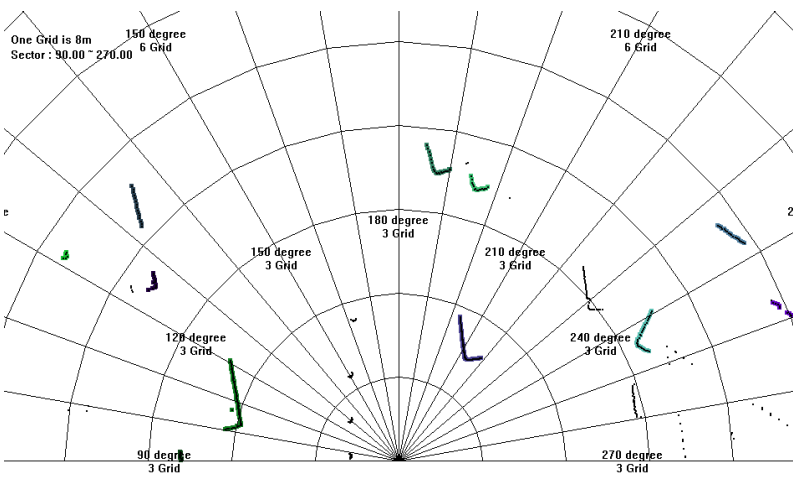

(a) 20th scan

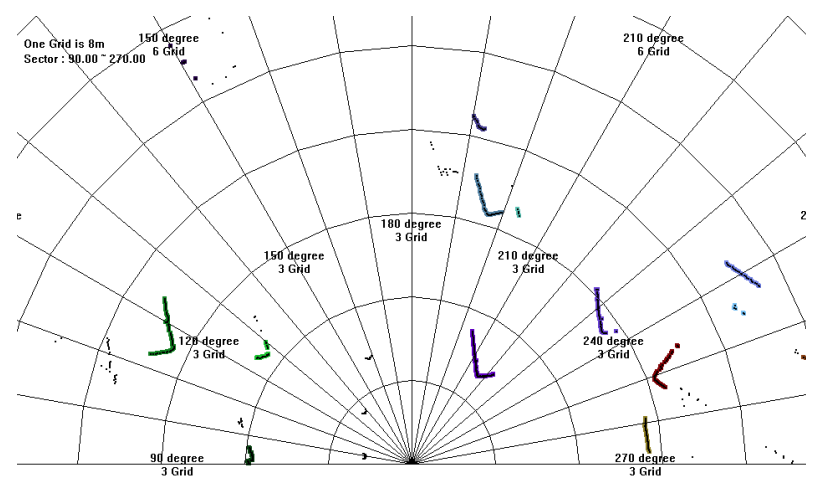

(b) 22th scan

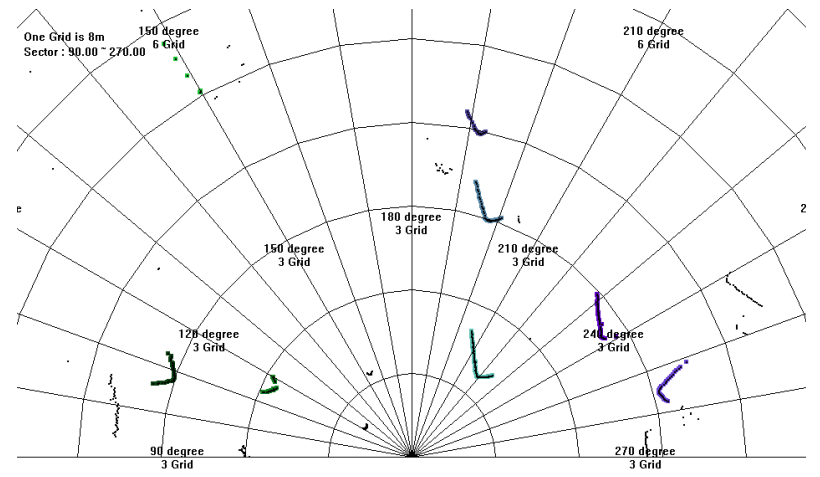

(c) 24th scan

Fig. 8 Results of detecting vehicles in the sequence of scans.
[6] K. Fuerstenberg, K. Dietmayer, V. Willhoeft, "Pedestrian Recognition in Urban Traffic using a vehicle based Multilayer Laserscanner," Proc. IV 2002, IEEE Intelligent Vehicles Symposium, pp. 31 35, 2002,

[7] D. Streller, K. Furstenberg, K. Dietmayer, "Vehicle and object models for robust tracking in traffic scenes using laser range images," Proc. Intelligent Transportation Systems, pp. 118 123, 2002.

[8] R. MacLachlan, C. Mertz, "Tracking of Moving Objects from a Moving Vehicle Using a Scanning Laser Rangefinder," Proc. Intelligent Transportation Systems, IEEE, pp. 301 306, 2006.

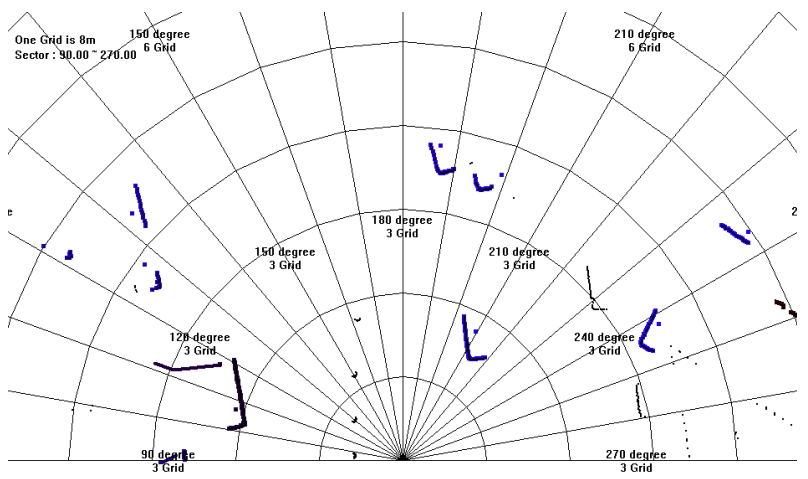

(a) 20th scan

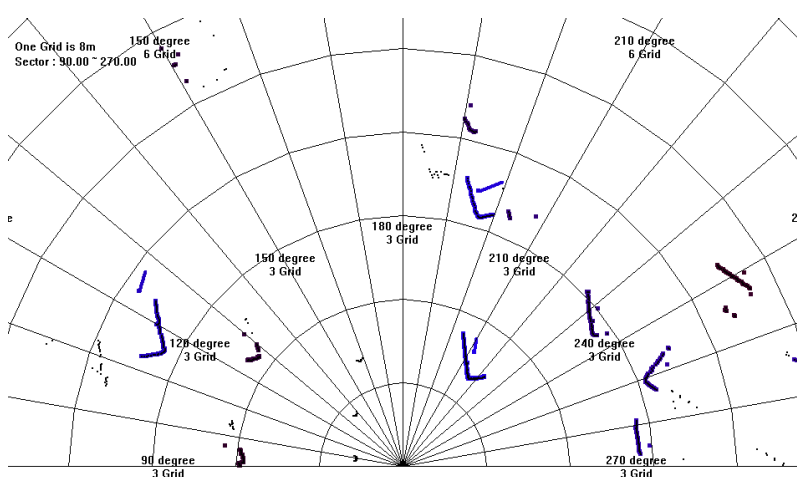

(b) 22th scan

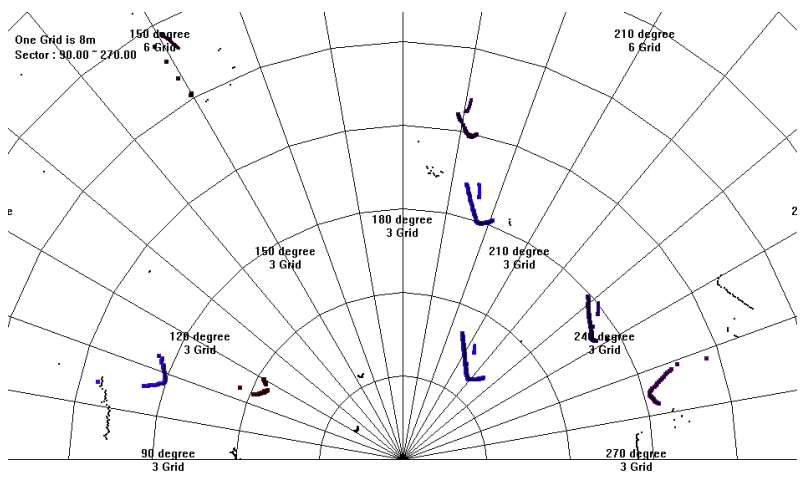

(c) 24th scan

Fig. 9 Results of multi-hypothesis tracking in the sequence of scans. 
[9] D. Streller, K. Dietmayer, J. Sparbert, "Object Tracking in Traffic Scenes with Multi-Hypothesis Approach using Laser Range Images," Proc. ITS 2001, 8th World Congress on Intelligent Transport Systems, Sydney, Australia, 2001.

[10] D. Streller, K. Dietmayer, "Object tracking and classification using a multiple hypothesis approach," Proc. Intelligent Vehicles Symposium, IEEE, pp. 808 812, 2004.

[11] D. Streller, K. Dietmayer, "Multiple hypothesis classification with laser range finders," Proc. Intelligent Transportation Systems, IEEE, pp. 195 200, 2004.

[12] Y. Obata, M. Ito, S. Tsujimichi, Y. Kosuge, "Computation-time Reduction of Track Oriented Multiple Hypothesis Tracking," Proc. the 40th SICE Annual Conference, pp. 282 287, 2001.

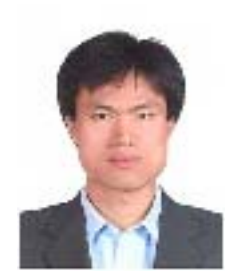

Kyungjin Ryu

was born in Youngju, Korea, in 1980. He received the B.S. and the M.S. degrees in electrical and electronic engineering, from Yonsei University, Seoul, Korea, in 2006 and 2008, respectively. His current research interests include intelligent vehicle system, tracking and state observer.

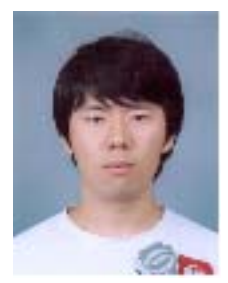

\section{Seongkeun Park}

was born in Seoul, Korea, in 1981. He received the B.S degree in electric and electronic engineering from Yonsei University, Seoul, Korea, in 2004 and he is currently in M.S. and PH.D. combined program in electric and electronic engineering at the same university. His current research interests focuses on state estimation, radar signal processing and intelligence vehicle system

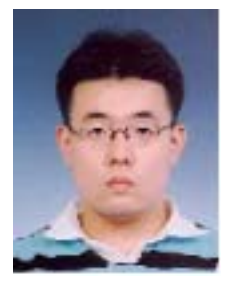

Jae Pil Hwang

was born in Seoul, Korea, in 1977. He received the B.S. and the M.S. degrees in electrical and electronic engineering, from Yonsei University, Seoul, Korea, in 2003 and 2005, respectively. He is currently a $\mathrm{Ph}$. D. candidate of Dept. of electrical and electronic engineering at Yonsei University. His current research interests include vision application for vehicles, computer vision and pattern recognition.

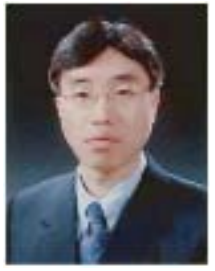

\section{Euntai Kim}

was born in Seoul, Korea, in 1970 . He received the B.S. (summa cum laude) and the M.S. and the Ph.D. degrees in electronic engineering, all from Yonsei University, Seoul, Korea, in 1992, 1994 and 1999, respectively. From 1999 to 2002, he was a full-time lecturer in the Department of Control and Instrumentation Engineering at Hankyong National University, Kyonggi-do, Korea. Since 2002, he has joined the faculties of the School of Electrical and Electronic Engineering at Yonsei University, where he is currently an associate professor. His current research interests include computational intelligence and its application to intelligent service robot and intelligent home network. Dr. Kim is an associate editor of International Journal of Control and Systems.

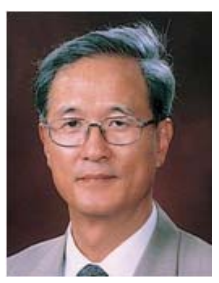

\section{Mignon Park}

received a B.S. and an M.S. in electronics from Yonsei University, Seoul, Korea, in 1973 and 1977, and a Ph.D. from the University of Tokyo, Tokyo, Japan, in 1982. He was a researcher in the Institute of Biomedical Engineering, University of Tokyo, from 1972 to 1982, as well as at the Massachusetts Institute of Technology, Cambridge, and the University of California Berkeley, in 1982. He was a visiting researcher in the Robotics Division, Mechanical Engineering Laboratory Ministry of International Trade and Industry, Tsukuba, Japan, from 1986 to 1987. He has been a Professor in the Department of Electrical and Electronic Engineering, Yonsei University, since 1982. His research interests include fuzzy control and applications, robotics, and fuzzy biomedical systems. 